DOI: $10.24850 /$ j-tyca-2019-03-10

Notas

\title{
Variabilidad climática y eventos extremos en la cuenca del río Zanatenco, Chiapas
}

\section{Climate variability and extreme events in the Zanatenco river basin, Chiapas}

José Luis Arellano-Monterrosas ${ }^{1}$

Laura Elena Ruiz-Meza²

${ }^{1}$ Comisión Nacional del Agua. Organismo de Cuenca Frontera Sur, México, jose.arellanoa@conagua.gob.mx

${ }^{2}$ Universidad de Ciencias y Artes de Chiapas. Instituto de Ciencias Biológicas, México, laura.ruiz@unicach.mx

Autor para correspondencia: Laura Elena Ruiz Meza, laura.ruiz@unicach.mx

\section{Resumen}

Se analiza la variabilidad climática interanual para el periodo de 1961 a 2013 en la cuenca del río Zanatenco, ubicada en la costa de Chiapas, México. Se analizaron las siguientes variables: precipitación y evaporación, tanto anual como máxima diaria, al igual que temperaturas medias y máximas. También se analizó la incidencia de eventos hidrometeorológicos extremos asociados con estas variables climatológicas, como las inundaciones y sequías. Las inundaciones están asociadas con los años con mayores precipitaciones, así como a la magnitud de los eventos de lluvia máxima diaria. Por su parte, las sequías se asocian con los años con menores precipitaciones, y con temperaturas máximas y tasas de evaporación diarias extremas. Los años secos y lluviosos están relacionados con la presencia de los fenómenos climáticos globales de El Niño y La Niña. La precipitación y evaporación anual, así como la temperatura media anual muestran una tendencia creciente. Los eventos extremos de precipitación diaria se 
presentaron de 2005 a 2013; los de evaporación de 2001 a 2008; y los de temperatura de 1997 a 2006, correspondientes a años con sequía. El año con el evento de lluvias extremas máximas registrado fue 2006. La mayor tasa de evaporación diaria se presentó en 1970 y, la mayor temperatura máxima se registró en los años de 1997 a 1998. De esta forma, a partir de 1997, las sequías e inundaciones se han presentado con mayor frecuencia e intensidad en la región.

Palabras clave: tendencias cambio climático, eventos extremos, inundaciones, sequías, costa de Chiapas.

\section{Abstract}

The interannual climatic variability is analyzed for the period 1961 to 2013 in the Zanatenco river basin, located in the Chiapas Coast, Mexico. The following variables were analyzed: precipitation and evaporation, both annual and daily maximum, as well as average and maximum temperatures. Likewise, the incidence of extreme hydrometeorological events associated to these climatological variables, such as floods and droughts, was analyzed. The floods are associated with greater rainfall the years, as well as the magnitude of the maximum daily rainfall events. The droughts are associated to the years with lower rainfall as well as extreme temperatures and extreme daily evaporation rates. The dry and rainy years are related to the presence of the global climatic phenomena of El Niño and La Niña. Annual precipitation and evaporation as well as the annual average temperature show an increasing trend. The extreme events of daily precipitation were presented from 2005 to 2013; the evaporation from 2001 to 2008; and of temperature from 1997 to 2006, corresponding to years with drought. The year with the maximum extreme rainfall event was recorded in 2006. The highest rate of daily evaporation occurred in 1970 and the highest maximum temperature occurred in the years 1997 to 1998. Thus, since 1997, droughts and floods have occurred more frequently and intensively in the región.

Keywords: Climate change trends, extreme events, floods, droughts, Chiapas Coast.

Recibido: 04/05/2017

Aceptado: 22/01/2019 


\section{Introducción}

En las últimas décadas, en los regiones donde las lluvias dependen en gran medida de la actividad del océano Pacífico (ciclones tropicales y huracanes), como en la costa de Chiapas, las tendencias en la variabilidad interanual de la lluvia son decrecientes (Martínez, 2007; Méndez \& Magaña, 2010; Magaña, Matías, Morales, \& Millán, 2004). Para Martínez, Patiño y Tamayo (2014), el análisis de las tendencias climáticas en una región debe realizarse mediante la revisión de los valores medios y extremos de las principales variables climáticas. Para Montealegre (2009), la variabilidad climática interanual corresponde a las variaciones que se presentan en las variables climatológicas año con año.

Desde un punto de vista estadístico, se define como evento extremo a aquel cuya probabilidad de ser igualado o superado es muy pequeña (Arganis, Domínguez, Jiménez, \& Guichard, 2010). En el caso de inundaciones y sequías, la principal dificultad está en establecer la magnitud del evento al que se refiere. Para los propósitos de este estudio, los eventos extremos de inundaciones y sequías están intrínsecamente relacionados con la magnitud del evento climático perturbador, pero también a las condiciones específicas de vulnerabilidad de la zona expuesta a su impacto. Según los escenarios de cambio climático del Panel Internacional de Cambio Climático (IPCC), se predicen en México cambios en el régimen hidrometeorológico, particularmente en la frecuencia de eventos extremos, como inundaciones y sequías (Magaña, 2004; Martínez, 2007).

En los últimos años, en las cuencas de la Sierra Madre y costa de Chiapas se presentan perturbaciones en el ciclo hidrológico regional, con tendencias decrecientes de la precipitación anual. Asimismo, los eventos de lluvias extremos diarias son cada más intensos y frecuentes (Arellano, 2010).

En el mapa de zonas de impactos potenciales por inundación de México, la costa de Chiapas presenta una frecuencia de inundaciones con 
periodos de recurrencia de 5 a 50 años. Asimismo, en el mapa de peligro por inundación, la región aparece con un índice alto por inundaciones (Semarnat, 2012).

Según Merz et al. (2014), en el enfoque tradicional hidrológico para la estimación de inundaciones, las crecientes son resultado de las características propias de la cuenca: geología, relieve, topografía, condiciones meteorológicas y suelos. De esta forma, la variabilidad natural del clima proporciona la variación en el tiempo de las características de las inundaciones; esta variación puede ser cuantificable y predecible bajo una perspectiva dinámica de clima y gestión de crecientes.

La lluvia es el elemento fundamental del ciclo hidrológico, que se define por su magnitud y distribución temporal, condiciones medias de humedad y anomalías anuales (excesos y déficits); la intensidad de la sequía se estima como un déficit de lluvia (Velasco, 2012). La sequía forma parte de la variabilidad natural del clima y es recurrente sin un ciclo definido. La magnitud de sus impactos depende de la vulnerabilidad de los sistemas naturales y sociales a esta condición (Hill et al., 2014). En las últimas décadas, el aumento de la vulnerabilidad hidrológica, agrícola y social ante la sequía se percibe en la región como un fenómeno cada vez más severo (Semarnat, 2012).

\section{Materiales y métodos}

\section{Zona de estudio}

La cuenca del río Zanatenco se ubica en el extremo noroeste de la Región Hidrológica 023 Costa de Chiapas (SRH, 1969a; SRH, 1969b), y forma parte de la Subregión Hidrológica Laguna del Mar Muerto. Geográficamente, se ubica entre las coordenadas $15^{\circ} 59^{\prime} 19^{\prime}$ ' y $16^{\circ}$ $09^{\prime} 34^{\prime \prime}$ ' de latitud norte, y $93^{\circ} 46^{\prime} 52^{\prime \prime}$ y $93^{\circ} 35^{\prime} 24^{\prime}$ ' de longitud 
oeste (Figura 1). Limita al noreste con su parteaguas en la Sierra Madre de Chiapas; al noroeste, con la cuenca del río Tiltepec; al sur, con la laguna del Mar Muerto, y al sureste con la cuenca del Riíto. Desde el parteaguas hasta su desembocadura en la laguna del Mar Muerto, la cuenca tiene una superficie total de $231.0552 \mathrm{~km}^{2}$. Administrativamente, la cuenca es parte de la región Istmo-Costa, en los municipios de Tonalá y Villaflores. En la parte media alta y alta de la cuenca se ubica parte de la Reserva de la Biosfera de la Sepultura (REBISE).
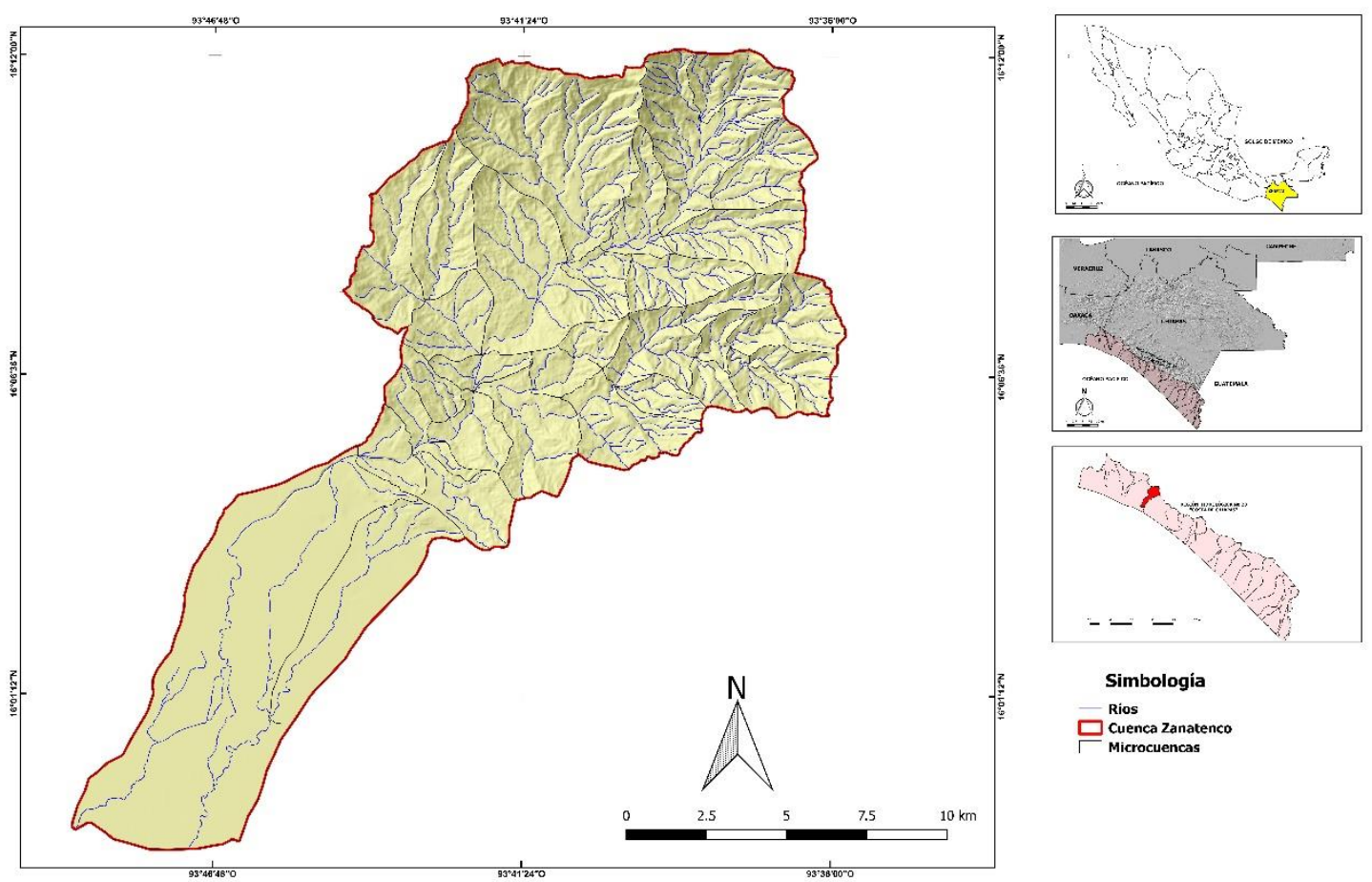

Figura 1. Ubicación de la cuenca del río Zanatenco, Chiapas. México. Fuente: elaboración propia.

La cuenca del río Zanatenco forma parte de las regiones fisiográficas de La Sierra del Sur o Sierra Madre de Chiapas en su parte media y alta (68.28\% de su territorio), y de la Llanura Costera del Pacífico en su parte baja $(31.72 \%)$. En la vertiente Pacífico de la Sierra Madre, el declive es muy pronunciado, por lo que los ríos son de régimen torrencial y cursos cortos. Por su parte, la planicie costera se ha 
conformado de modo superficial, como resultado de la constante depositación de sedimentos provenientes de la erosión de la Sierra Madre (Müllerried, citado por Conanp, 1999). En la cuenca alta, los suelos son derivados de rocas ígneas extrusivas, con profundidades mayores de $60 \mathrm{~cm}$. En la planicie costera se presentan suelos de origen aluvial, caracterizados por su textura arcillo-arenosa y depósitos arenosos, con profundidades mayores de $200 \mathrm{~cm}$, con fluctuaciones del nivel freático, donde los perfiles de suelos moteados son indicadores de periodos de inundaciones estacionales.

En la cuenca baja, el tipo de clima corresponde al $\mathrm{A}\left(W_{2}\right)$ : cálido subhúmedo con temperaturas máximas de $37^{\circ} \mathrm{C}$, temperatura media anual mayor de $34^{\circ} \mathrm{C}$ y temperatura del mes más frío mayor de $33^{\circ} \mathrm{C}$. La precipitación del mes más seco es menor de $1 \mathrm{~mm}$, con lluvias en verano y precipitaciones máximas de $400 \mathrm{~mm}$ en el mes de septiembre (Comisión Nacional del Agua, Universidad de Ciencias y Artes de Chiapas, Instituto Estatal del Agua y Ayuntamiento de Tonalá, 2012).

Para el periodo de registro de 1961 a 2013 en la estación climatológica Tonalá, Chiapas, la precipitación media anual es de $1723.8 \mathrm{~mm}$; la evaporación media anual de $2297.6 \mathrm{~mm}$, y la temperatura media anual de $28.7^{\circ} \mathrm{C}$. El periodo de lluvias comprende de mayo a octubre $(95.7 \%$ del promedio anual), con presencia de un periodo de sequía intraestival o canícula en el mes de julio. El periodo de secas o estiaje comprende los meses de noviembre a abril, con un $4.3 \%$ del promedio anual. La precipitación máxima mensual se presenta en septiembre, con 416.9 $\mathrm{mm}$, y la mínima mensual en diciembre con $1.3 \mathrm{~mm}$. La temperatura promedio anual es de $28.7^{\circ} \mathrm{C}$, la máxima mensual de $34.8^{\circ} \mathrm{C}$ y la mínima mensual de $22.7^{\circ} \mathrm{C}$.

En 2011, la superficie con bosques y selvas representó un $46.6 \%$ de la cuenca del río Zanatenco; los pastizales, un 41.1\%; la agricultura, un $12 \%$, y un $0.3 \%$ los asentamientos humanos.

\section{Análisis de información climatológica e hidrométrica}


Se considera a la estación climatológica Tonalá, Chiapas, como representativa de la cuenca baja del río Zanatenco debido a su ubicación y a que cuenta con el mayor registro histórico de información en la región. La estación climatológica Tonalá se ubica a $16^{\circ} 05^{\prime}$ de latitud norte y $93^{\circ} 42^{\prime}$ de longitud oeste, a una elevación de $17 \mathrm{msnm}$ (Serrano et al., 2006). Para el análisis de la información del río Zanatenco se utilizaron los registros de la estación hidrométrica Zanatenco, ubicada a $16^{\circ} 05^{\prime}$ de latitud norte, y $93^{\circ} 42^{\prime}$ de longitud oeste (SRH, 1969a; SRH, 1969b).

Se obtuvieron los datos diarios de precipitación, temperaturas (máxima, media, mínima), y evaporación para el periodo de 1961 a 2010 con uso de la base de datos ERIC III v. 3.2 (IMTA, 2013), misma que se complementó con datos proporcionados por el Centro Hidrometeorológico Regional de Conagua ubicado en Tuxtla Gutiérrez, Chiapas. La información histórica de gastos máximos de escurrimiento del río Zanatenco se obtuvo de la base de datos Bandas (Conagua, 2010).

Los datos se operaron en hojas de Excel. Se calcularon los parámetros estadísticos descriptivos de las variables climatológicas de estudio: medias, valores máximos, mínimos, rango, desviación estándar, coeficiente de variación. La variación interanual se analizó mediante regresión lineal (Yevdjevich, 1964; Infante \& Zárate, 1984), para obtener su tendencia, tasa de cambio anual y su coeficiente de correlación $R^{2}$. Para el cálculo de la precipitación y evaporación anual, así como de la temperatura media anual, sólo se analizaron años con registros completos, no se estimaron datos faltantes. Los parámetros estadísticos y las gráficas de dispersión permitieron el análisis de la calidad de la información y depuración de datos (Yevdjevich, 1964). Para el ajuste de datos hidrométricos a diferentes funciones probabilísticas se aplicó el programa $A X$, desarrollado por Jiménez, Domínguez y Cruz (1997).

\section{Resultados y discusión}




\section{Variabilidad de la precipitación anual}

Los años lluviosos corresponden a los siguientes: 1963, 1968, 1973, 1974, 1980, 1981, 1988, 1989, 1995, 1998, 2005, 2006, 2007 у 2013. El año 2005 es más lluvioso del registro histórico, con una precipitación anual de $2801.1 \mathrm{~mm}$. Para el periodo 1964-2003, Imbach, Pérez, Ulloa y Díaz (2013) reportan para la estación Tonalá, Chiapas, los siguientes años como los más lluviosos: 1980, 1981, 1989, 1993 y 1995, que corresponden a los reportados en este trabajo. Por otra parte, los años con menor precipitación anual corresponden a 1975, 1976, 1977, 1983, 1987, 1991, 1994 y 2001 . El año más seco del periodo fue 1987, con 1 $101.3 \mathrm{~mm}$.

La precipitación mensual acumulada máxima se presenta durante agosto, septiembre y octubre, con $651.3,982.5$ y $712.5 \mathrm{~mm}$, ocurridos en los años de 1969, 1963 y 2005, respectivamente. En promedio, los meses más lluviosos son los de junio y septiembre, con precipitaciones medias de 328.7 y $416.9 \mathrm{~mm}$, respectivamente, que corresponden a las lluvias de verano y la presencia de ciclones tropicales en la región. Serrano et al. (2006) reportan como los más lluviosos para su periodo de estudio (1961-1998): junio con $323.9 \mathrm{~mm}$ y septiembre con $399.4 \mathrm{~mm}$. Por su parte, Imbach et al. (2013) reportan valores de precipitaciones mensuales mayores de $800 \mathrm{~mm}$ en agosto y septiembre de 1988 y 1989, años asociados con periodos de la Oscilación del Sur El Niño (ENSO). Los valores de precipitación máxima mensual correspondientes a los meses de agosto y septiembre son de 651.3 y $982.5 \mathrm{~mm}$, respectivamente.

Se obtiene una tendencia general creciente en la precipitación anual con una tasa de $+3.61 \mathrm{~mm} / a n ̃$, con una correlación muy baja $\left(R^{2}\right.$ de 0.02$)$, misma que muestra la gran variabilidad de los datos sin una tendencia bien definida (Figura 2), como la reportada por Imbach et al. (2013). Por su parte, Escalante (2006), con información anterior a las inundaciones de septiembre de 1998, reporta también para la precipitación anual una tendencia estable. 


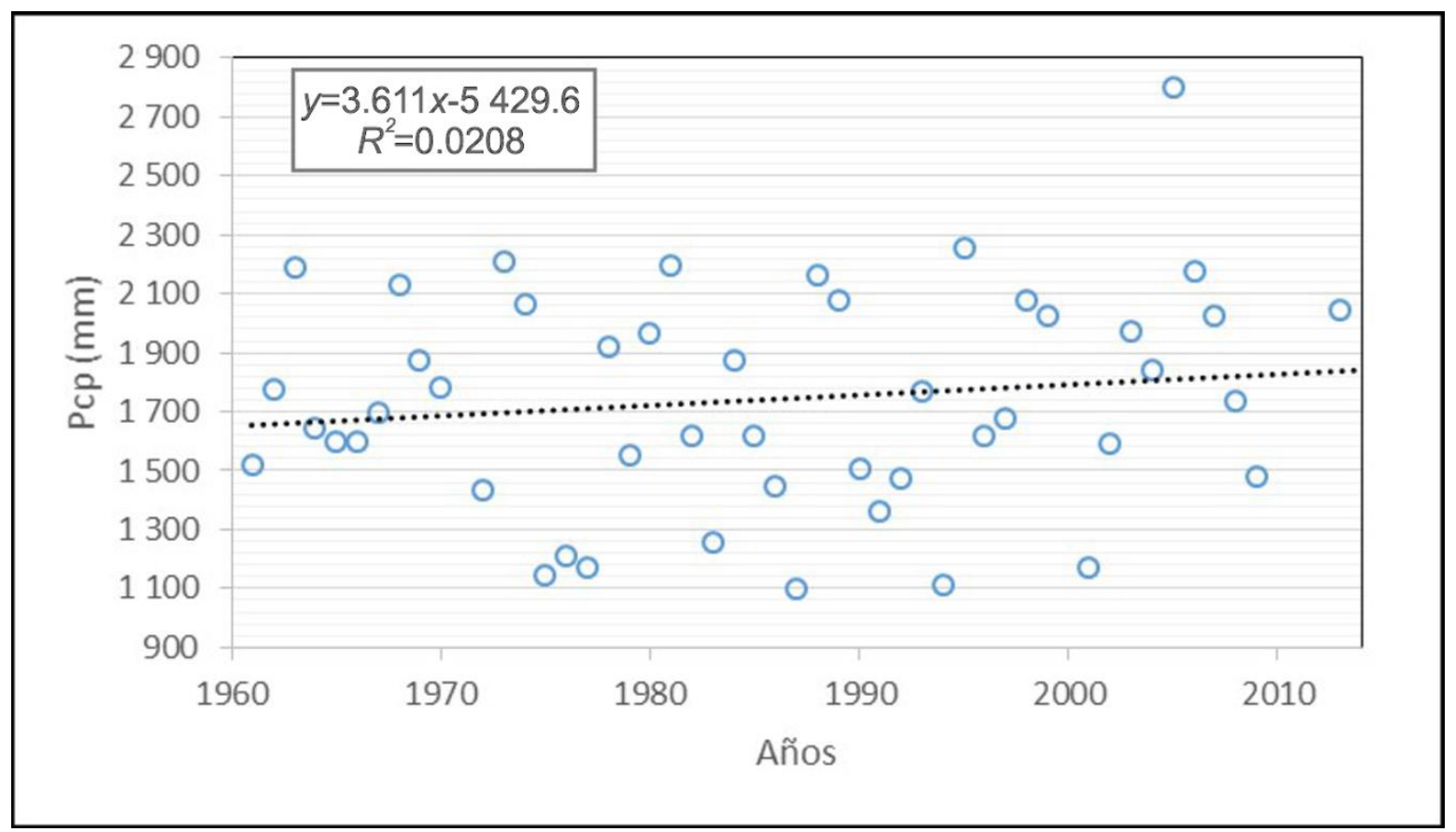

Figura 2. Variabilidad de la precipitación anual ( $\mathrm{mm}$ ) en Tonalá, Chiapas (1961-2013). Fuente: elaboración propia.

Las tendencias de variabilidad de la precipitación interanual en la costa de Chiapas muestran el patrón general reportado por el IPCC para el Pacífico mexicano. En las zonas más lluviosas (como el Soconusco), las tendencias en la precipitación son decrecientes, mientras que en las zonas menos lluviosas (como la región Istmo Costa), las tendencias son crecientes (Magaña et al., 2004).

Según Méndez y Magaña (2010), la precipitación anual en la región Istmo-Costa de Chiapas para el periodo 1901-2009 muestra

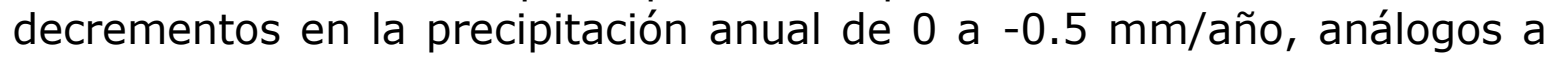
los reportados por Semarnat (2012). Por su parte, Martínez (2007) estima para el periodo 2070-2099 un cambio porcentual en la precipitación en la costa de Chiapas de -20 a $-40 \%$ en invierno y de 0 a $+20 \%$ en verano.

\section{Variabilidad de la temperatura media anual}


La temperatura media promedio anual muestra los siguientes periodos decrecientes: 1961-1968, 1969-1971, 1972-1986 y 2003-2009, así como los siguientes periodos crecientes: 1968-1969, 1971-1972, 19862003.

La variabilidad interanual de la temperatura media anual muestra una tendencia general creciente a una tasa de $0.0121{ }^{\circ} \mathrm{C} / \mathrm{año}$, con un coeficiente de correlación $\left(R^{2}\right)$ de 0.1189 , que equivale a un incremento promedio de $0.58{ }^{\circ} \mathrm{C}$ para el periodo de estudio de 48 años (Figura 3). Este incremento, se encuentra en el rango reportado por el Gobierno del Estado de Chiapas et al. (2011), para el periodo 1951-2000 de hasta 0.4 ${ }^{\circ} \mathrm{C} / a n ̃ o$, al igual que en el rango reportado por Méndez y Magaña (2010) para la costa de Chiapas de 0.5 a $1.0^{\circ} \mathrm{C} / \mathrm{año}$.

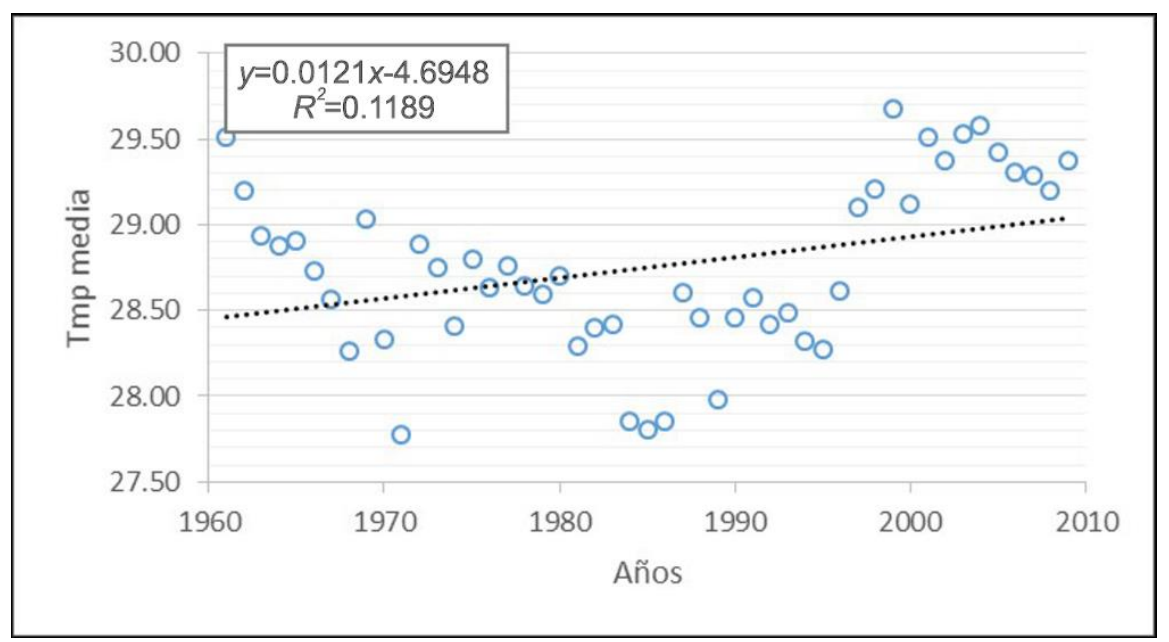

Figura 3. Variabilidad de la temperatura media anual $\left(\mathrm{C}^{\circ}\right)$ en Tonalá, Chiapas (1961-2013). Fuente: elaboración propia.

De seguir esta tendencia de crecimiento en la temperatura media anual, para los años 2050 y 2100 , se esperarían incrementos en la temperatura máxima de 0.6 y $1.2{ }^{\circ} \mathrm{C}$, respectivamente, valores que se encuentran por abajo del escenario de 2 a $4{ }^{\circ} \mathrm{C}$ para finales del siglo XXI propuesto por Méndez y Magaña (2010). 
En el estado de Tabasco, Rivera et al. (2016) obtienen también para el periodo 1961-2010 una tendencia general creciente en la temperatura promedio anual con una tasa de $0.0171^{\circ} \mathrm{C} / a n ̃ o$, casi la misma tasa que la obtenida para Tonalá, Chiapas, lo cual muestra cierta tendencia regional en el sur de México.

\section{Variabilidad de la evaporación anual}

La evaporación anual máxima se presentó en 2001 con 2505.24 mm y la mínima en 1962 con $1569.6 \mathrm{~mm}$. Para el periodo de estudio (19612002), se obtiene una marcada tendencia creciente en la variabilidad interanual de la evaporación a una tasa de $+11.35 \mathrm{~mm} / a n ̃ o$, con un buen coeficiente de correlación $R^{2}$ de 0.4495 (Figura 4). Para la región IstmoCosta de Chiapas, Martínez (2007) estima una evapotranspiración potencial anual de $640 \mathrm{~mm}$, e incrementos en la temperatura máxima de 0.6 y $1.2^{\circ} \mathrm{C}$ en Tonalá, Chiapas, para 2050 y 2100 , respectivamente. Asimismo, con incrementos en la temperatura de 1 a $3{ }^{\circ} \mathrm{C}$, estima incrementos de evapotranspiración potencial anual de $40 \mathrm{~mm}$. Una tendencia creciente en la evaporación anual implica mayor demanda de agua para el desarrollo de los cultivos (Ojeda, Sifuentes, Íñiguez, \& Montes, 2011). 


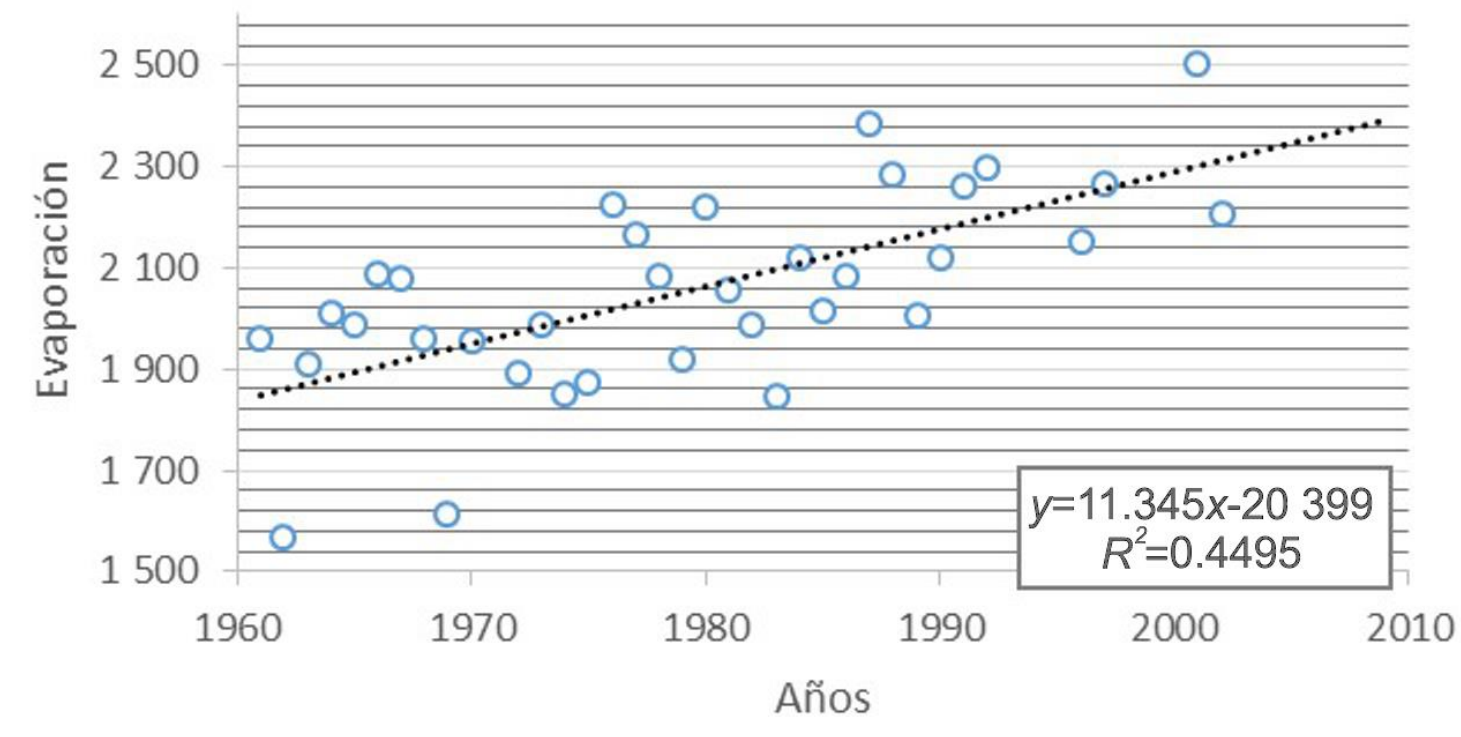

Figura 4. Variabilidad de la evaporación anual $(\mathrm{mm})$ en Tonalá, Chiapas (1961-2013). Fuente: elaboración propia.

\section{Eventos hidrometeorológicos extremos: inundaciones y sequías}

Los años que presentaron los eventos máximos diarios de precipitación fueron 1963, 1980, 2005, 2006 y 2013; los de evaporación máxima diaria: 1970, 1993, 2001, 2003, 2005 y 2008; y los de temperatura máxima diaria: 1962, 1969, 1997, 1998, 2002, 2004 y 2006 (Tabla 1).

Tabla 1. Eventos climatológicos extremos diarios en Tonalá, Chiapas

(1961-2013). Fuente: elaboración propia.

\begin{tabular}{|c|c|c|c|c|c|c|c|c|}
\hline Año & $\begin{array}{c}\text { Prec. } \\
(\mathbf{m m})\end{array}$ & $\begin{array}{c}\text { Evaporación } \\
(\mathbf{m m})\end{array}$ & $\begin{array}{c}\text { Temperatura } \\
\left({ }^{\circ} \mathbf{C}\right)\end{array}$ & & Año & $\begin{array}{c}\text { Prec. } \\
(\mathbf{m m})\end{array}$ & $\begin{array}{c}\text { Evaporación } \\
(\mathbf{m m})\end{array}$ & $\begin{array}{c}\text { Temperatura } \\
\left({ }^{\circ} \mathbf{C}\right)\end{array}$ \\
\hline 1961 & 96.0 & 10.8 & 41.0 & & 1986 & 107.1 & 14.8 & 39.0 \\
\hline 1962 & 139.0 & 12.1 & 42.0 & & 1987 & 75.8 & 15.1 & 40.5 \\
\hline 1963 & 290.0 & 13.1 & 41.5 & & 1988 & 214.8 & 15.6 & 41.0 \\
\hline
\end{tabular}




\begin{tabular}{|r|c|c|c|c|c|c|c|c|}
\hline 1964 & 111.0 & 13.6 & 41.0 & & 1989 & 208.5 & 16.1 & 40.0 \\
\hline 1965 & 90.0 & 13.8 & 40.5 & & 1990 & 75.1 & 12.7 & 39.0 \\
\hline 1966 & 93.0 & 15.8 & 41.0 & & 1991 & 105.3 & 13.5 & 39.0 \\
\hline 1967 & 148.0 & 15.0 & 41.5 & & 1992 & 82.2 & 15.0 & 42.0 \\
\hline 1968 & 115.0 & 16.0 & 39.5 & & 1993 & 171.7 & 17.2 & 40.0 \\
\hline 1969 & 125.8 & 16.3 & 42.0 & & 1994 & 85.0 & 14.3 & 39.5 \\
\hline 1970 & 96.8 & 17.5 & 41.5 & & 1995 & 230.6 & 13.8 & 40.5 \\
\hline 1971 & 66.0 & N/D & 38.5 & & 1996 & 112.6 & 15.1 & 40.5 \\
\hline 1972 & 88.2 & 15.3 & 41.0 & & 1997 & 165.2 & 14.9 & 42.5 \\
\hline 1973 & 151.0 & 14.0 & 41.0 & & 1998 & 180.8 & 15.4 & 42.5 \\
\hline 1974 & 142.8 & 14.4 & 39.0 & & 1999 & 96.0 & 13.6 & 38.1 \\
\hline 1975 & 54.2 & 12.6 & 41.5 & & 2000 & 92.1 & 16.2 & 39.5 \\
\hline 1976 & 79.8 & 13.2 & 41.0 & & 2001 & 57.1 & 16.7 & 41.5 \\
\hline 1977 & 84.0 & 15.7 & 41.0 & & 2002 & 136.4 & 15.8 & 42.0 \\
\hline 1978 & 82.9 & 15.3 & 40.1 & & 2003 & 115.4 & 17.4 & 41.5 \\
\hline 1979 & 74.8 & 15.6 & 40.5 & & 2004 & 90.9 & 13.3 & 42.5 \\
\hline 1980 & 257.5 & 14.3 & 41.0 & & 2005 & 269.6 & 16.7 & 41.0 \\
\hline 1981 & 217.1 & 14.3 & 40.5 & & 2006 & 312.7 & 14.1 & 42.0 \\
\hline 1982 & 125.4 & 14.5 & 39.5 & & 2007 & 144.8 & 14.9 & 41.0 \\
\hline 1983 & 76.0 & 12.2 & 40.0 & & 2008 & 125.4 & 17.0 & 41.5 \\
\hline 1984 & 149.6 & 15.1 & 39.0 & & 2009 & 128.0 & $\mathrm{~N} / \mathrm{D}$ & 41.0 \\
\hline 1985 & 95.4 & 16.0 & 40.0 & & 2013 & 255.0 & $\mathrm{~N} / \mathrm{D}$ & $\mathrm{N} / \mathrm{D}$ \\
\hline & & & & & & & & \\
\hline
\end{tabular}

\section{Variabilidad de la precipitación máxima diaria}

Los eventos de precipitación máxima diaria registrados fueron de 312.7 $\mathrm{mm}$ en 2006, de $290.0 \mathrm{~mm}$ en 1963, y de $269.6 \mathrm{~mm}$ en 2005 con el ciclón tropical Stan (Tabla 1). Por su parte, Escalante (2006) reporta para la estación Tonalá, Chiapas, como los años con lluvias más severas a 1963 y 1998, con láminas acumuladas en diez días cercanas a los 800 $\mathrm{mm}$. En su análisis de precipitaciones diarias, en periodos de diez años para la misma estación, Imbach et al. (2013) reportan valores de precipitación diaria mayor de $150 \mathrm{~mm}$ (de $257.5 \mathrm{~mm}$ en 1980); sin embargo, en su estudio quedaron fuera años muy lluviosos: 2005, 2006 y 2013, con precipitaciones máximas diarias mayores de $260 \mathrm{~mm}$ 
asociadas con el ciclón tropical Stan de octubre de 2005, y las tormentas tropicales Bárbara de mayo de 2013, e Ingrid y Manuel de septiembre del mismo año.

Se obtiene una tendencia general creciente en la variabilidad interanual

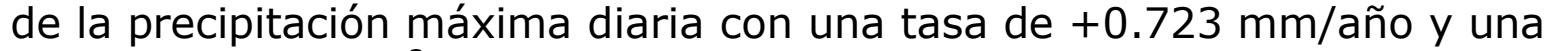
baja correlación $\left(R^{2}=0.027\right)$, con una gran variabilidad en los datos sin una tendencia bien definida (Figura 5 ). Para la precipitación máxima diaria, la función de mejor ajuste corresponde a la doble Gumbel, con un error estándar de 0.407 . Las precipitaciones máximas diarias como las de 1963 de 290.0 mm o las máximas históricas registradas de 2006 con $312.7 \mathrm{~mm}$ corresponden a periodos de retorno $(\mathrm{Tr})$ de alrededor de 50 años (Tabla 2).

Tabla 2. Precipitaciones máximas diarias en Tonalá, Chiapas, ajustadas a la función Gumbel para diferentes periodos de retorno ( $T r)$. Fuente: elaboración propia.

\begin{tabular}{|c|c|}
\hline $\begin{array}{c}\text { Tr } \\
\text { (años) }\end{array}$ & $\begin{array}{c}\text { Prec. máx. 24 } \\
\text { horas (mm) }\end{array}$ \\
\hline 2 & 113.0 \\
\hline 5 & 181.5 \\
\hline 10 & 245.3 \\
\hline 20 & 275.7 \\
\hline 50 & 309.3 \\
\hline 100 & 333.1 \\
\hline
\end{tabular}




\section{Ciencias $₫$ Agua}

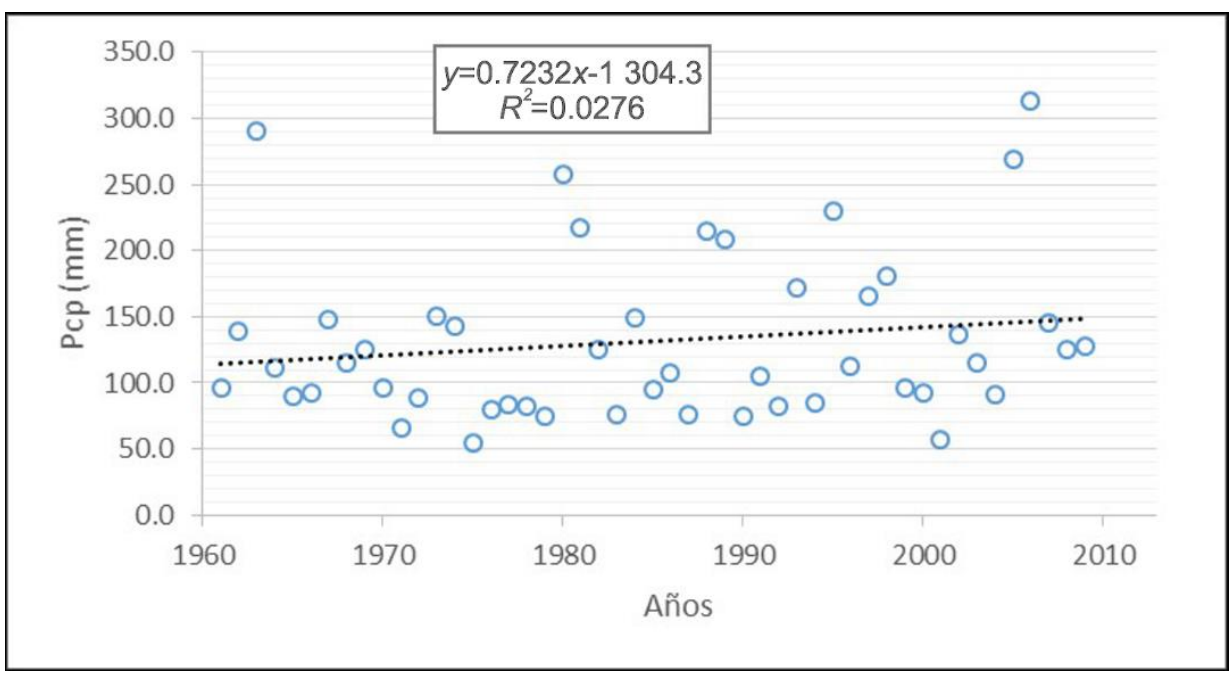

Figura 5. Variabilidad de la precipitación máxima anual ( $\mathrm{mm}$ ) en Tonalá, Chiapas (1961-2013). Fuente: elaboración propia.

\section{Gastos máximos de escurrimiento en el río Zanatenco}

Los años con escurrimientos máximos en el río Zanatenco corresponden a 1963, 1966, 1975 y 1989. Para el río Zanatenco, la función de mejor ajuste corresponde a la Log Normal, con un error estándar de 46.974. El gasto máximo de escurrimiento se registró el 23 de septiembre de 1963 con $741.163 \mathrm{~m}^{3} / \mathrm{s}$ (SRH, 1969a; SRH, 1969b; Conagua, 2010), mismo que corresponde a un periodo de retorno ( $T r$ ) cercano a los 100 años (Tabla 3).

Tabla 3. Gastos máximos de escurrimiento ajustados a la función Log Normal para diferentes periodos de retorno (Tr) en el río zanatenco, Chiapas. Fuente: elaboración propia.

\begin{tabular}{|c|c|}
\hline $\begin{array}{c}\boldsymbol{T r} \\
\text { (años) }\end{array}$ & $\begin{array}{c}\text { Gastos } \\
\text { máximos } \\
\left(\mathbf{m}^{\mathbf{3}} / \mathbf{s}\right)\end{array}$ \\
\hline 2 & 90.27 \\
\hline 5 & 201.03 \\
\hline
\end{tabular}




\begin{tabular}{|c|c|}
\hline 10 & 305.50 \\
\hline 20 & 275.70 \\
\hline 50 & 431.64 \\
\hline 100 & 825.78 \\
\hline
\end{tabular}

En su estudio hidrológico de la costa de Chiapas, Domínguez et al. (2006) incorporaron las estimaciones de gasto de escurrimiento provocado por el ciclón tropical Stan de octubre de 2005, con lo cual obtienen para el río Zanatenco los siguientes resultados: gasto de escurrimiento máximo (Qmáx) de $741.2 \mathrm{~m}^{3} / \mathrm{s}$; gasto de escurrimiento medio (Qmed) de $158.5 \mathrm{~m}^{3} / \mathrm{s}$; desviación estándar de 142.5, y coeficiente de variación de 0.90 . Estos datos indican que el gasto de escurrimiento máximo estimado por el impacto de Stan en el río Zanatenco fue menor al registrado en septiembre de 1963; los autores ajustaron los datos a una función doble Gumbel para diferentes periodos de retorno $(T r)$.

Los valores obtenidos en este trabajo son similares a los reportados por Domínguez et al. (2006), en particular para los periodos de retorno de 5 a 100 años, utilizados por lo general en el diseño de obras hidráulicas de protección contra inundaciones (Conagua, 2011; Ordaz, Torres, \& Domínguez, 2013).

\section{Inundaciones}

El impacto de los eventos hidrometeorológicos de septiembre de 1998 y del ciclón tropical Stan fueron particularmente significativos en la región. Ambos fenómenos evidenciaron el grado de vulnerabilidad de las comunidades de la Sierra Madre y costa de Chiapas (Arellano, 2010), en sobre todo de las comunidades costeras de la planicie, así como las de pescadores del municipio de Tonalá (Cuevas, 2009). De 2010 a 2013 se presentaron diez fenómenos climáticos en la región. Tales eventos están relacionados con el fenómeno de La Niña de 2010 y 2013, considerados de los años más lluviosos de la Sierra Madre de Chiapas. 


\section{Variabilidad de la temperatura diaria máxima}

Los eventos históricos de temperaturas máximas varían de 38.5 a 42.5 ${ }^{\circ} \mathrm{C}$ (Figura 6). Las temperaturas diarias máximas mayores de $42{ }^{\circ} \mathrm{C}$ se presentaron en 1962, 1969, 1997, 1998, 2002, 2004 y 2006 (Tabla 1).

A partir de 1997, los eventos de temperaturas máximas asociadas con sequías se han tenido con mayor frecuencia e intensidad en la región (Figura 6). Los eventos extremos de temperatura diaria de 1962 se asocian con el fenómeno de El Niño de 1962-1963, 1969, 1997-1998 y 2005.

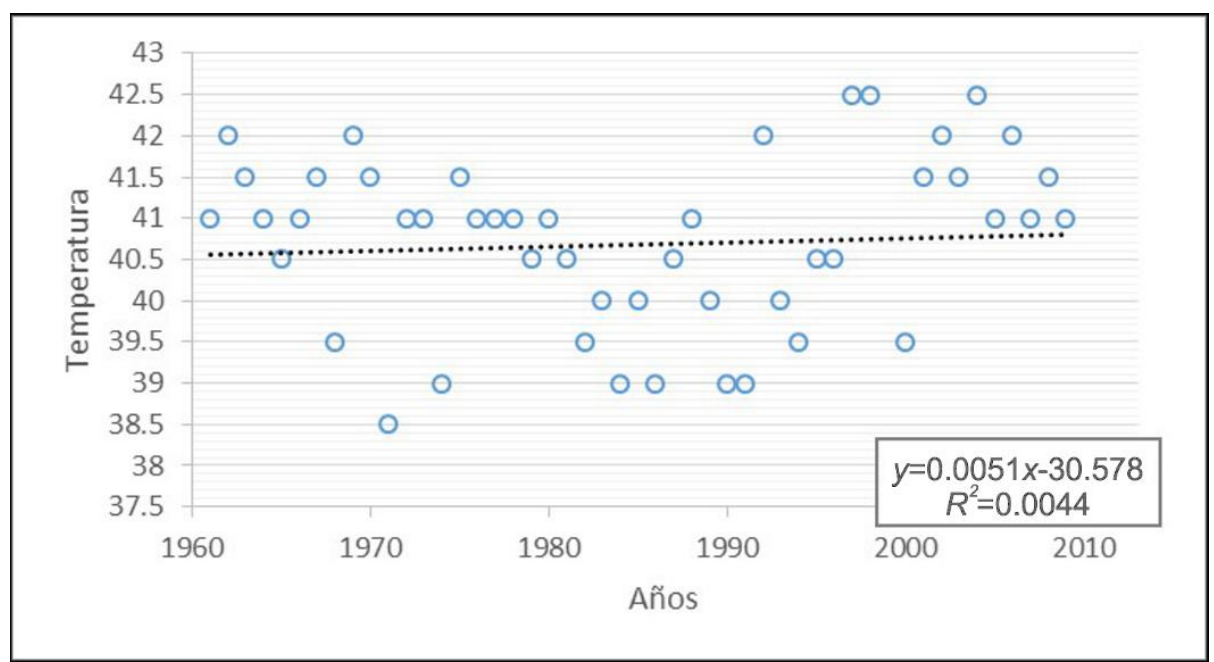

Figura 6. Temperatura extremas máximas diarias $\left({ }^{\circ} \mathrm{C}\right)$ en Tonalá, Chiapas (19612009). Fuente: elaboración propia.

Imbach et al. (2013) reportan para el periodo de 1964-2003 valores de temperatura máxima mayores de $42{ }^{\circ} \mathrm{C}$ en febrero. Las temperaturas máximas superiores a los $41.5^{\circ} \mathrm{C}$ no se presentaron de 1970 a 1992. Debido a que el valor de $R^{2}$ del modelo lineal de regresión es muy bajo $\left(R^{2}=0.0044\right)$, los datos muestran una gran dispersión; sin embargo, se obtiene una tendencia creciente de $0.0051^{\circ} \mathrm{C} / \mathrm{año}$. De seguir esta 
tendencia, en los próximos 100 años se tendría un incremento de 0.51 ${ }^{\circ} \mathrm{C}$ (Figura 6). Por su parte, De la Mora et al. (2016) obtienen oscilaciones con tendencias crecientes en la temperatura máxima extrema, con temperaturas mayores a $38^{\circ} \mathrm{C}$, con promedios móviles de cuatro años, donde reportan temperaturas extremas mayores a $38^{\circ} \mathrm{C}$ en 1986,2003 y 2009.

\section{Evaporación máxima diaria}

Los eventos máximos de evaporación diaria se presentaron en 1970, 1983, 1993, 2001, 2003, 2005 y 2008 (Tabla 1). La variabilidad interanual de la evaporación máxima diaria es creciente, con una tasa de $0.036 \mathrm{~mm} /$ año y una $R^{2}=0.1155$ (Figura 7).

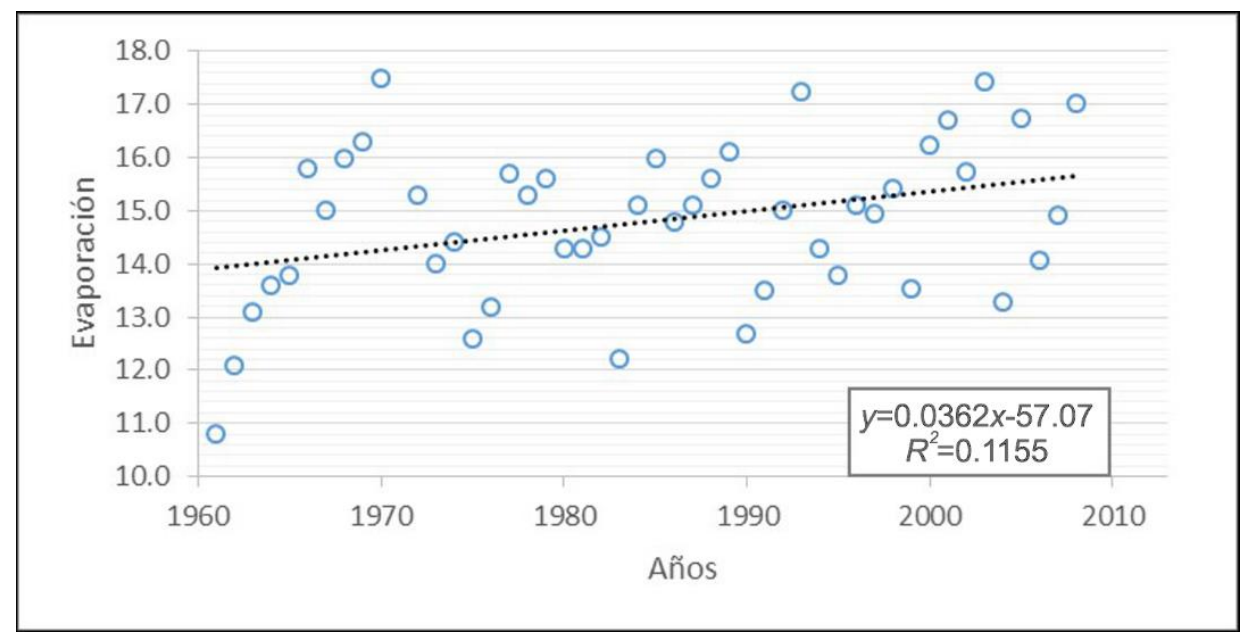

Figura 7. Evaporación máxima diaria $\left({ }^{\circ} \mathrm{C}\right.$ ) en Tonalá, Chiapas (19612009). Fuente: elaboración propia.

A partir de 2001, los eventos de evaporación máxima diaria asociadas con sequías se han presentado con mayor frecuencia e intensidad en la región. Los eventos extremos de evaporación diaria de 1983 se relacionan con El Niño 1982-1983 y la de 2005 con El Niño de ese mismo año. Es importante anotar que la evaporación máxima no 
necesariamente se presenta los días de máxima temperatura; la evaporación depende también de la radiación solar, humedad relativa y el viento.

\section{Sequías}

La incidencia de sequía se vincula con las precipitaciones que se presentan por debajo de la media histórica en periodos de 2 a 10 años. Según los registros históricos de lluvia, para la estación de Tonalá, Chiapas, los años secos por debajo de $1200 \mathrm{~mm}$ fueron los de 1975, 1977, 1987, 1994 y 2001.

Según el estudio de Conagua (2014) en Tonalá, Chiapas, en el periodo de 1961-2008, se han presentado 46 eventos de sequía moderada, 44 de sequía severa y seis de sequía extrema. Los eventos de sequía extrema se presentaron en 1971, 2000 y 2001. Sin embargo, los años de 1971 y 2000 reportados también en el estudio de Conagua (2014) con sequía extrema no cuentan con registros históricos completos de Iluvia. Asimismo, Imbach et al. (2013) indican que en 2000, la estación Tonalá no tiene datos de lluvia completos, por lo que sólo reportan sequía extrema en 2001.

En su estudio de sequía realizado en el país, Velasco (2012) señala para la costa de Chiapas anomalías de lluvia anual que indican sequía en 1991, así como los periodos de sequía de 1992 a 1994, y de 1998 a 2002. Para Tiscareño et al. (1998), los años de 1963, 1965, 1969, 1972, 1982, 1987 y 1997 fueron años secos, con presencia del fenómeno El Niño. Según Magaña et al. (2004), los años El Niño de 1982-1983 y 1997-1998 fueron de lo más extremos en el siglo XX.

Los registros de lluvia corroboran como año seco 1987 (1 $101.3 \mathrm{~mm}$ ) asociado con el fenómeno El Niño de ese año; 2001 (1 $168.1 \mathrm{~mm})$, con el periodo seco del índice SPI de 1998-2002, asociado con El Niño de 1997-1998. Los años 2005 y 2010 fueron también de transición El NiñoLa Niña, es decir, muy secos y muy lluviosos.

La sequía de 1997 a 1998 fue tan grave que se presentaron enormes pérdidas en la agricultura e impactos en 198808 hectáreas de incendios 
forestales en Chiapas; El Niño de 1997 a 1998 fue el evento climático más severo del periodo 1982-2000 en Chiapas, mismo que provocó un incremento significativo de las temperaturas máximas y el déficit de precipitación, con alteraciones en el ciclo hidrológico y, en consenciencia, de disponibilidad de agua (Magaña et al., 2004; Gobierno del Estado de Chiapas et al., 2011).

En la región Istmo-Costa, las temperaturas extremas del periodo 19971998 fueron las máximas registradas: $42.5^{\circ} \mathrm{C}$. De junio a octubre de 1997 se estimaron anomalías de precipitación de 10 a 50\% por abajo del promedio de lluvia del cuatrimestre (Tiscareño et al., 1998). El periodo de estiaje de 1998 fue particularmente deficitario en precipitación en Chiapas. Meses más tarde, debido a la transición del fenómeno El Niño a La Niña, se presentaron lluvias extremas en la región Sierra Madre y costa de Chiapas, que provocaron la contingencia hidrometeorológica de septiembre de 1998, con los impactos más severos que se han registrado en la historia reciente en la región Istmo-Costa.

Según los registros hidrométricos disponibles del río Zanatenco, en 11 de los 30 años de registro considerados en el periodo de estudio de 1963 a 2009 (lo que representa 33\%), uno de casi cada tres años, el río se ha secado varios días en 1969, 1973, 1976-1978, 1982-1985, 1989, 2003-2008. Asimismo, se registraron gastos mínimos muy pequeños de un litro por segundo en 1979 y 1980.

En la costa de Chiapas, de enero a abril de 2016, se presentó una anomalía de precipitación de 50 a $75 \%$; es decir, por debajo de la media histórica para los mismos meses (Conagua, 2016). En diferentes testimonios, la población local refiere como años particularmente secos 1973, 1998, 2002, 2015 y la temporada de secas 2016.

\section{Conclusiones}

Los años secos y lluviosos guardan relación con la presencia de periodos de El Niño y La Niña en la región. Para el periodo 1961-2013, la variabilidad de la precipitación anual muestra una tendencia general 
creciente, con una tasa de $+3.61 \mathrm{~mm} /$ año. La temperatura media anual presenta una tendencia general creciente a una tasa de $0.0121{ }^{\circ} \mathrm{C}$ por año, lo cual equivale a un incremento promedio de $0.58{ }^{\circ} \mathrm{C}$ para el periodo de estudio de 48 años. De igual forma, la evaporación anual muestra una marcada tendencia creciente, con una tasa de +11.35 $\mathrm{mm} / a$ ño.

Las inundaciones están asociadas con los años con mayores precipitaciones, así como con los eventos de lluvia máxima diaria. Las sequías se relacionan con los años con menores precipitaciones, así como con temperaturas máximas y tasas de evaporación diarias extremas.

El año con el evento de lluvias extremas máximas diarias fue 2006, con $312.7 \mathrm{~mm}$ (periodo de La Niña). La mayor tasa de evaporación diaria extrema se presentó en 1970 (17.5 mm), comprendido entre los años de El Niño 1969 y 1972. La mayor temperatura máxima se registró en 1997 y 1998 , con $42.5^{\circ} \mathrm{C}$ (periodo de El Niño).

Los eventos extremos diarios de precipitación se presentaron en el periodo de 2005 a 2013; los de evaporación de 2001 a 2008, y los de temperatura de 1997 a 2006. Entonces, a partir de 1997, los eventos extremos de precipitación están asociados con inundaciones, así como los de evaporación y temperaturas lo están con sequías. Lo anterior coincide con la presencia de eventos climáticos extremos: precipitaciones de 2005 a 2013; evaporación de 2001 a 2008, y temperatura de 1997 a 2006.

Los eventos de temperaturas máximas vinculadas con sequías también se han presentado con mayor frecuencia e intensidad en la región. Los eventos extremos de temperatura de 1962 se relacionan con El Niño 1962-1963; el evento de 1969, con el fenómeno de El Niño de ese año; los de 1997-1998, con el fenómeno de El Niño de ese periodo, y las temperaturas máximas de 2004 y 2006 se asocian con El Niño de 2005.

Los registros de lluvia corroboran como año seco 1987 (1 $101.3 \mathrm{~mm}$ ) relacionado con el fenómeno de El Niño, así como 2001 (1 $168.1 \mathrm{~mm}$ ) con el periodo seco del SPI de 1998-2002 y con El Niño de 1997-1998. En el río Zanatenco, en 11 de los 30 años de registro (1963 a 2009), 1 de cada 3 años, el río se ha secado durante varios días.

\section{Agradecimientos}


Agradecemos al Programa de Investigación en Cambio Climático de la UNAM el apoyo para realizar la investigación de la que se derivó este artículo.

\section{Referencias}

Arellano, J. L. (2010). Gestión integral de recursos hídricos para reducir la vulnerabilidad a deslizamientos e inundaciones en las cuencas de la Sierra Madre de Chiapas. Ingeniería Agrícola y Biosistemas, 2(1), 29-37.

Arganis, M., Domínguez, R., Jiménez, M., \& Guichard, D. (2010). Eventos extremos. En: Jiménez, B., Torregrosa, M., \& Aboites, L. (eds.). El agua en México: cauces y encauces (pp. 563-594). México, DF, México: Academia Mexicana de Ciencias, Comisión Nacional del Agua.

Conagua, Comisión Nacional del Agua. (2010). Banco Nacional de Datos de Aguas Superficiales (Bandas). México, DF, México: Subdirección General Técnica, Gerencia de Aguas Superficiales e Ingeniería de Ríos (GASIR).

Conagua, Comisión Nacional del Agua. (2011). Manual para el control de inundaciones. México, DF, México: Comisión Nacional del Agua.

Conagua, Comisión Nacional del Agua. (2014). Programa de Medidas Preventivas y de Mitigación de la Sequía en la Cuenca de la Costa de Chiapas. Informe final. Tuxtla Gutiérrez, México: Organismo de Cuenca Frontera Sur, Universidad de Ciencias y Artes de Chiapas.

Conagua, Comisión Nacional del Agua. (2016). Pronóstico climatológico y condiciones hidrometeorológicas en la región. Presentación en la $10^{a}$ Sesión Ordinaria del Consejo de Cuenca de la Costa de Chiapas, Tapachula, Chiapas, 20 de mayo de 2016.

Comisión Nacional del Agua, Universidad de Ciencias y Artes de Chiapas, Instituto Estatal del Agua y Ayuntamiento de Tonalá. (2012). Plan de Gestión Integral de la cuenca del río Tiltepec, Chiapas. Tonalá, México: Comisión Nacional del Agua, Universidad de Ciencias y Artes de Chiapas, Instituto Estatal del Agua y Ayuntamiento de Tonalá.

Conanp, Comisión Nacional de Áreas Naturales Protegidas. (1999). Programa de Manejo Reserva de la Biósfera La Sepultura. México, 
DF, México: Secretaría de Medio Ambiente, Recursos Naturales y Pesca, Comisión Nacional de Áreas Naturales Protegidas.

Cuevas, J. (2009). Vivir el desastre. Análisis de la vulnerabilidad frente a inundaciones. El caso de una comunidad de pescadores en Chiapas. En: Vera, G. (coord.). Devastación y éxodo. Memoria de Seminario sobre Reubicaciones por Desastres en México (pp. 215227). México, DF, México: Centro de Investigaciones y Estudios Superiores en Antropología Social, Secretaría de Desarrollo Social, Consejo Nacional de Ciencias y Tecnología.

De la Mora, O. C., Ruíz, C. J., Flores, L. H., Zarazúa, V. P., Ramírez, O. G., Medina, G. G., Rodríguez, M. V., \& Chávez, D. A. (2016). Índices de cambio climático en el estado de Chiapas, México, en el periodo 1960-2009. Revista Mexicana de Ciencias Agrícolas, Pub. Esp. (13), 2523-2534.

Domínguez, M. R., Carrizosa, E. E., Fuentes, M. G., López, E. J., Herrera, A. J., \& Echeverri, V. C. (2006). Estudio para definir las obras de control contra inundaciones en las cuencas altas de los ríos del estado de Chiapas. Estudio hidrológico. Informe final (Proyecto 6307). México, DF, México: Instituto de Ingeniería, Universidad Nacional Autónoma de México para la Comisión Nacional del Agua.

Escalante, C. (2006). Análisis del patrón de lluvias en la costa de Chiapas. Quehacer Científico en Chiapas, 1(1), 39-64.

Gobierno del Estado de Chiapas, Secretaría de Medio Ambiente y Recursos Naturales, Embajada Británica, Instituto Nacional de Ecología, El Colegio de la Frontera Sur, Colegio de Posgraduados, Conservación Internacional. (2011). Programa de Acción ante el Cambio Climático del Estado de Chiapas. Tuxtla Gutiérrez, Chiapas: Secretaría de Medio Ambiente e Historia Natural.

Hill, H., Hadarits, M., Rieger, R., Strickert, G., Davies, E., \& Strobbe, K. (2014). The invitational drought tournament: What is it and why is it a useful tool for drought preparedness and adaptation? Weather and Climate Extremes. Recuperado de http.//dx.doi.org/10.1016/j.wace.2014.03.002

Imbach, A., Pérez, J., Ulloa, D., \& Díaz, Á. (2013). Análisis de la variabilidad climática en la costa de Chiapas, México. Tuxtla Gutiérrez, México : Centro Agronómico Tropical de Investigación y Enseñanza, The Nature Conservancy, Ministerio Federal de Medio Ambiente de Alemania. 
IMTA, Instituto Mexicano de Tecnología del Agua. (2013). ERIC III. Extractor rápido de información climática. Ver 3.2. Jiutepec, México: Instituto Mexicano de Tecnología del Agua.

Infante, S., \& Zárate, G. (1984). Métodos estadísticos. Un enfoque interdisciplinario. México, DF, México: Editorial Trillas.

Jiménez, M., Domínguez, R., \& Cruz, M. (1997). Manual de operación de los programas AX.AXE y CARACHID.EXE. México, DF, México: Centro Nacional de Prevención de Desastres.

Magaña, V. (2004). El cambio climático global: comprender el problema. En: Martínez, J., \& Fernández, A. (comp.). Cambio climático: una visión desde México (pp. 19-27). México, DF, México: Secretaría de Medio Ambiente y Recursos Naturales, Instituto Nacional de Ecología.

Magaña, V., Matías, J., Morales, R., \& Millán, C. (2004). Consecuencias presentes y futuras de la variabilidad y el cambio climático en México. En: Martínez, J., \& Fernández, A. (comp.). Cambio climático: una visión desde México (pp. 203-213). México, DF, México: Secretaría de Medio Ambiente y Recursos Naturales, Instituto Nacional de Ecología.

Martínez, P. (2007). Efectos del cambio climático en los recursos hídricos de México. Jiutepec, Morelos: Secretaría de Medio Ambiente y Recursos Naturales, Instituto Mexicano de Tecnología del Agua.

Martínez, P., Patiño, C., \& Tamayo, J. (2014). Análisis de tendencias climáticas en la cuenca baja del río Yaqui, Sonora, México. Tecnología y ciencias del agua, 5(6), 135-149.

Méndez, J., \& Magaña, R. (2010). Regional aspects of prolosced meteorological droughts over Mexico. Journal Climate, 23, 11751188.

Merz, B., Aerts, J., Arnbjerg, K., Baldi, M., Becker, A., Bichet, A., Bloschi, G., Bouwer, I. M., Brauer, A., Cioffi, I. F., Delgado, J. M., Gocht, M., Guzzetti, F., Harrigan, S., Hirschboock, K., Kilsby, C., Kron, W., Kron, H. H., Lall, U., Merz, R., Nissen, K., Salvatti, P., Swierczynski, T., Ulbrich, U., Viglione, A., Ward, P. J., Weiler, M., Wilhelm, B., \& Nied, M. (2014). Floods and climate: Emerging perspectives for flood risk assessment and management. Natural Hazards and Earth System Sciences, 14, 1921-1942. 
Montealegre, J. (2009). Estudio de la variabilidad climática de la precipitación en Colombia asociada a procesos oceánicos y atmosféricos de meso y gran escala. Informe final. Bogotá, Colombia: Instituto de Hidrología, Meteorología y Estudios Ambientales.

Ojeda, W., Sifuentes, E., Íñiguez, M., \& Montes, M. (2011). Impacto del cambio climático en el desarrollo y requerimientos hídricos de los cultivos. Agrociencia, 45, 1-11.

Ordaz, M., Torres, M., \& Domínguez, R. (2013). Vulnerabilidad y riesgo por inundaciones. México, DF, México: Colegio de Ingenieros Civiles de México, A. C., Instituto de Ingeniería de la UNAM y Carso Infraestructura y Construcciones.

Rivera, H. B., Aceves, N. L., Arrieta, R. A., Juárez, L. J., Méndez, A. J., \& Ramos, A. C. (2016). Evidencias del cambio climático en el estado de Tabasco durante el periodo 1961-2010. Revista Mexicana de Ciencias Agrícolas. Pub. Esp. (14), 2645-2656.

Semarnat, Secretaría de Medio Ambiente y Recursos Naturales. (2012). México. Quinta Comunicación Nacional ante la Convención Marco de las Naciones Unidas sobre el Cambio Climático. México, DF, México: Comisión Intersecretarial de Cambio Climático, Instituto Nacional de Ecología y Cambio Climático.

Serrano, V., Díaz, G., López, A., Cano, M., Báez, A., \& Garrido, E. (2006). Estadísticas climatológicas básicas del estado de Chiapas (periodo 1961-2003) (libro técnico núm. 1). Ocozocoautla de Espinosa, Chiapas, México: Campo Agrícola Experimental Centro de Chiapas del Instituto Nacional de Investigaciones Forestales, Agrícolas y Pecuarias.

SRH, Secretaría de Recursos Hidráulicos. (1969a). Boletín Hidrológico, (23), Costa de Chiapas.

SRH, Secretaría de Recursos Hidráulicos. (1969b). Boletín Hidrológico, (29), Coatzacoalcos.

Tiscareño, L. M., Rosenberg, N. J., Legler, D. M., Ruíz, C. A., Shrnvasan, R., Brown, R. A., García, M. G., Velázquez, V. M., \& Izurralde, C. (1998). Algunos efectos del fenómeno El Niño en la agricultura mexicana. Ciencia y Desarrollo, 25(139), 3-12.

Velasco, I. (2012). Sequía y cambio climático en México. Jiutepec, México: Instituto Mexicano de Tecnología del Agua. 
Yevdjevich, Y. M. (1964). Statistical and probability analysis of hydrologic data. Part II. Regression and correlation analysis. Section 8-II. En: Chow, V. T. (ed.). Handbook of applied hydrology. A compendium of water-resources technology (pp. 4367). New York, USA: McGraw-Hill Book Company. 